\title{
Exclusive Vector Meson Production at HERA
}

\author{
Arik Kreisel $^{1} 2$ \\ for the $\mathrm{H} 1$ and the ZEUS Collaborations \\ School of Physics and Astronomy, \\ Raymond and Beverly Sackler Faculty of Exact Sciences \\ Tel Aviv University, Tel Aviv, Israel.
}

\begin{abstract}
.
An extended study of exclusive vector meson production in $e p$ interactions has been performed by the H1 and the ZEUS collaborations at the HERA collider. Recent measurements are reported and discussed within the framework of the dipole model and $\mathrm{pQCD}$.
\end{abstract}

\section{INTRODUCTION}

The sharp rise of the electromagnetic proton structure function, $F_{2}$, toward low values of Bjorken $x$, discovered at HERA [1,2], and the observation of a large fraction of diffractive-like events $[3,4]$ are attributed to a large gluon density in the proton at very low $x$ values, typically $x<0.01$. The rise of $F_{2}$ with decreasing $x$ can be accommodated by the QCD, DGLAP evolution equations [5] in NLO down to momentum transfer squared $Q^{2} \simeq 1 \mathrm{GeV}^{2}[6,7]$. This suggests that perturbative effects set in at relatively low values of the interaction scale. However, the validity of the DGLAP evolution equation is established through fits to data which involve many unknown parameters and therefore it may be doubtful. Exclusive vector meson $(\mathrm{V})$ production at high $Q^{2}$ has been proposed as an alternative method to infer the gluon content of the proton [8].

High energy elastic V production in Deep Inelastic Scattering (DIS) may be described, in the rest frame of the proton, by the fallowing sequence of happenings [9]. The incoming lepton emits a virtual photon, which subsequently fluctuates into a $q \bar{q}$ pair. The life time of such a quark pair fluctuation is long enough so that it is the pair that elastically scatters off the proton and evolves, long after the interaction, into a $\mathrm{V}$ state.

1) Email: arikk@alzt.tau.ac.il

2) LISHEP 2002 - Session C:Workshop On Diffractive Physics - February 4 - 82002 Rio de Janeiro - RJ - Brazil 
The character of the interaction of the $q \bar{q}$ pair with the proton depends on the transverse momenta of the pair. If the transverse momentum is large, the spatial transverse separation between the quarks is small and it forms a color dipole, whose interaction with the proton may be calculated perturbatively [10]. The leading process is two gluon exchange. If the transverse momentum is small, the color dipole is large and perturbative calculations do not apply. In this case the interaction looks similar to hadron-hadron elastic scattering and the process should proceed through Pomeron exchange as expected from Regge phenomenology [11].

The $q \bar{q}$ wave function of the virtual photon depends on the polarization of the virtual photon. For longitudinally polarized photons, small transverse size $q \bar{q}$ dominate. The opposite is true for transversely polarized photons. The attractive features of elastic $\mathrm{V}$ production is that, at high $Q^{2}$, the longitudinal component of the virtual photon dominates. The interaction cross section for the latter can be, in principle, fully calculated in perturbative QCD. Moreover, for heavy vector mesons, like the $J / \Psi$ or the $\Upsilon$, perturbative calculations apply even at $Q^{2}=0$, as the smallness of the dipole is guaranteed by the mass of the quarks.

Independently of particular calculations $[12,13]$, in the region dominated by perturbative QCD, the following features are predicted:

- the total $\gamma^{\star} p \rightarrow V p$ cross section, $\sigma_{\gamma^{\star} p \rightarrow V p}$, exhibits a steep rise with $W$, the photon-proton center-of-mass energy, that can be parameterized as $\sigma \sim W^{\delta}$, with $\delta$ increasing with $Q^{2}$;

- the $Q^{2}$ dependence, which for a longitudinally polarized photon is expected to behave like $Q^{-6}$, becomes milder, more like $Q^{-4}$, due to the sharp increase of the gluon density with $Q^{2}$;

- the distribution of $t$, the four momentum transfer squared at the proton vertex, becomes universal, with little or no dependence on $W$ or $Q^{2}$;

All these features have been observed at HERA [14-17] in the exclusive production of $\rho^{0}, \phi, \omega$ and $J / \Psi$ mesons. However, the agreement between measurements and theory is far from perfect. There are many factors that may spoil the agreement. First and foremost, the measured cross sections contain the possibly soft transverse component of the virtual photon, which has to be modeled. In addition, the perturbative calculations have the following uncertainties:

- the calculation of $\sigma_{\gamma^{\star} p \rightarrow V p}$ involves the so-called skewed parton distributions [18], which are not yet well tested and involve gluon distributions outside the range which is constrained by global parton density analyses [19];

- higher order corrections have not been fully calculated, therefore the overall normalization is uncertain and the scale at which the gluons are probed is not known;

- the fast rise of $\sigma_{\gamma^{\star} p \rightarrow V p}$ implies the presence of the real part of the scattering amplitude, which is not fully known; 
- the wave functions of the vector mesons are not fully under control.

In spite of all these problems, it is generally felt that precise measurements of $\sigma_{\gamma^{\star} p \rightarrow V p}$ as a function of $W, Q^{2}, t$ and the mass of the vector meson, $M_{V}$, with a separation into longitudinal and transverse components [20], will help to resolve the theoretical uncertainties and ultimately lead to a better understanding of the parton distributions in the proton as well as of the dynamics of high energy interactions in the presence of a large scale.

One of the challenges in confronting perturbative QCD calculations with data is the ability to establish a region where hard interactions dominate over the soft component. The soft component is believed to be well described by Regge phenomenology, according to which at high energy, the Pomeron exchange dominates in the production of diffractive states. The parameters of the Pomeron trajectory are known from measurements of total cross sections in hadron-hadron interactions and elastic proton-proton measurements. It is usually assumed that the Pomeron trajectory is linear in $t$ and has the following form:

$$
\alpha_{\mathbb{P}}(t)=\alpha_{\mathbb{P}}(0)+\alpha_{\mathbb{P}}^{\prime} t
$$

The parameter $\alpha_{\mathbb{P}}(0)$ determines the energy behavior of the total cross section,

$$
\sigma_{\text {tot }} \sim s^{\alpha_{\mathbb{P}}(0)-1}
$$

and $\alpha_{\mathbb{P}}^{\prime}$ describes the increase of the exponential slope $b$ of the $t$ distribution with increasing $s$. The value of $\alpha_{\mathbb{P}}^{\prime}$ is expected to be inversely proportional to the typical transverse momenta squared of the partons participating in the exchanged trajectory [21]. Indeed a large value of $\alpha_{\mathbb{P}}^{\prime}$ would suggest the presence of low transverse momenta, typical of soft interactions. The latest fits of $\alpha_{\mathbb{P}}(0)[22]$ and $\alpha_{\mathbb{P}}^{\prime}[23]$ are give:

$$
\begin{aligned}
\alpha_{\mathbb{P}}(0) & =1.096 \pm 0.003 \\
\alpha_{\mathbb{P}}^{\prime} & =0.25 \mathrm{GeV}^{-2} .
\end{aligned}
$$

These values for the trajectory parameters describe the so called soft Pomeron trajectory. The non-universality of $\alpha_{\mathbb{P}}(0)$ has been established in DIS, where the slope of the rise of the $\gamma^{\star} p$ total cross section with $W$ has a pronounced $Q^{2}$ dependence $[24,25]$. The issue of the $\alpha_{I P}^{\prime}$ dependence on the hardness of the interaction may be addressed in the study of exclusive $\mathrm{V}$ production at HERA. The value of $\alpha_{\mathbb{P}}^{\prime}$ may be determined from the $W$ dependence of $b$, since $b$ is expected to behave as

$$
b(W)=b_{0}+4 \alpha_{\mathbb{P}}^{\prime} \ln \frac{W}{W_{0}} .
$$

The parameter $\alpha_{\mathbb{P}}^{\prime}$ may also be derived from the $W$ dependence of the differential cross section $d \sigma / d t$ at fixed $t$, 


$$
\frac{d \sigma}{d t}(W)=F(t) W^{2\left[2 \alpha_{\mathbb{P}}(t)-2\right]}
$$

where $F(t)$ is a function of $t$ only. The latter approach has the advantage that no assumption needs to be made about the $t$ dependence. The measurements of $\alpha_{\mathbb{P}}(t)$ in exclusive $J / \psi$ photoproduction show that, while $\alpha_{\mathbb{I P}}(0)$ for $J / \psi$ is larger the soft $\alpha_{\mathbb{P}}(0)$ value, the value of $\alpha_{\mathbb{P}}^{\prime}$ for $J / \psi$ is smaller $[16,26,27]$ than the soft value.

Another way to investigate the contribution of hard and soft components in $\mathrm{V}$ production is by projecting out the interactions induced by the longitudinally and transversely polarized virtual photons. Due to the respective wave functions structure, small $q \bar{q}$ configurations are dominantly longitudinal while large configurations are dominantly transverse. If one assumes that exclusive $\mathrm{V}$ production proceeds through $s$-channel helicity conservation ( $\mathrm{SCHC}$ ), the separation into the longitudinal and transverse components is possible. At small $t$, the hypothesis of SCHC has been directly tested in the data, and small deviations have been observed $[28,29]$. However, the deviations are small enough not to jeopardize the decomposition of the cross sections into the longitudinal and transverse components.

The angular distributions of the decay products of the vector mesons give access to the spin density matrix elements, which are bilinear combinations of the helicity amplitudes $T_{\lambda_{V} \lambda_{\gamma}}$ where $\lambda_{V}\left(\lambda_{\gamma}\right)$ is the $\mathrm{V}$ (virtual photon) helicity [30]. As $t$ increases, a possible change in helicity is more likely due to the change in the V's direction and the transfer of the transverse momentum carried by the gluons. The following features are expected by pQCD models $[13,31,32]$ in DIS for $|t| \lesssim Q^{2}$ :

- a constant ratio of the helicity conserving amplitudes, $\left|T_{11}\right| /\left|T_{00}\right|$, with $t$;

- a $\sqrt{|t|}$ dependence for the ratio of the single helicity flip to the non-flip amplitudes $\left|T_{01}\right| /\left|T_{00}\right|$ and $\left|T_{10}\right| /\left|T_{00}\right|$;

- a linear $t$ dependence for the ratio of the double flip to the non-flip amplitudes $\left|T_{1-1}\right| /\left|T_{00}\right|$;

- the hierarchy

$$
\left|T_{00}\right|>\left|T_{11}\right|>\left|T_{01}\right|>\left|T_{10}\right|>\left|T_{1-1}\right|
$$

The differential cross section, $\frac{d \sigma}{d t}$, of $\mathrm{V}$ production has an exponentially falling cross section at low $t$ and a power like behavior at high $t$. An exponential behavior is associated with a Gaussian charge distribution and the slope, $b$, of the exponent is related to the width of the distribution and is a measure of the interaction size. In a naive picture for elastic scattering, $b$ has contributions from the size of the $q \bar{q}$ pair which decreases with $Q^{2}$ and the constant size for the proton. At high $Q^{2}$, for all type of elastic $\mathrm{V}$ production, $b$ should reach an asymptotic value equal to the proton size.

The cross section for elastic $\mathrm{V}$ photo or electroproduction, with small transfer momentum to the proton, has the form [33] 


$$
\frac{d \sigma_{\gamma p \rightarrow V p}}{d t}=F_{p}^{2}(t) F_{V}^{2}\left(t, Q^{2}\right)\left|A\left(W, t, Q^{2}\right)\right|^{2},
$$

where $F_{p}(t)$ and $F_{V}(t)$ are the form factors of the proton and the V, respectively, that account for the probabilities of their elastic production, and $A(W, t)$ is the amplitude of the constituent interaction. The cross section of proton dissociation $\mathrm{V}$ production does not contain the proton form factor

$$
\frac{d \sigma_{\gamma p \rightarrow V Y}}{d t}=F_{V}^{2}\left(t, Q^{2}\right)\left|A\left(W, t, Q^{2}\right)\right|^{2} .
$$

Vertex factorization [34] predicts that the ratio of proton dissociation over elastic differential cross section is $Q^{2}$ independent. The ratio of equation (8) to equation (9) is equal to the square of the proton form factor.

\section{II $W$ DEPENDENCE OF V PRODUCTION}

The dependence of the cross section $\sigma_{V}=\sigma_{\gamma^{(\star)} p \rightarrow V p}$ on the center of mass energy, $W$, is shown in Fig. 1 for photoproduction of various vector mesons [35]. While the $W$ dependence of light vector mesons $(\rho \omega \phi)$ is $\sigma_{V}(W) \propto W^{0.22}$, as expected from Regge phenomenology, the $J / \psi$ cross section has a steep rise with $W$, which is a signature of a hard process, as predicted by pQCD.

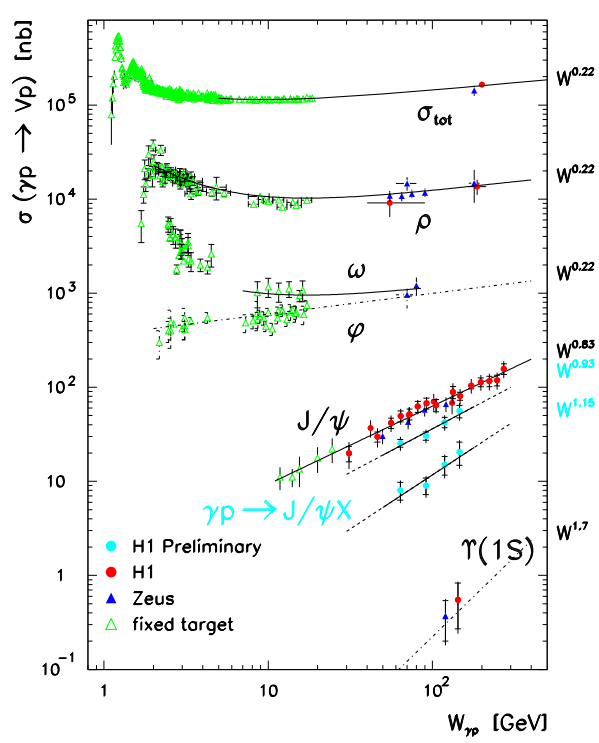

FIGURE 1. The photoproduction cross section as a function of $W$, for different vector mesons. The lines show a $W$ dependence, with $\delta$ values as indicated.

A change in the $W$ dependence is also seen when moving from low to high $Q^{2}$, as shown in Fig. 2, where the $W$ dependence of $\sigma_{\rho}$ for various $Q^{2}$ values [28] is 
plotted . For each $Q^{2}$ value the $W$ dependence of $\sigma_{\rho}$ is fitted with a form $W^{\delta}$. The parameter $\delta$ is related to the exchanged trajectory $\alpha_{\mathbb{P}}(t)$ by $\left.\delta=4(\alpha(<t\rangle)-1\right)$. To extract $\alpha_{\mathbb{P}}(0)$ from $\delta,<|t|>=1 / b$ is taken from measured values [28] and the value $\alpha_{P}^{\prime}=0.25 \mathrm{GeV}^{-2}$ is assigned. The resulting $\alpha_{\mathbb{P}}(0)$ is plotted as a function of $Q^{2}$ in Fig. 3 and a marked increase of $\alpha_{\mathbb{P}}(0)$ with $Q^{2}$ is observed.

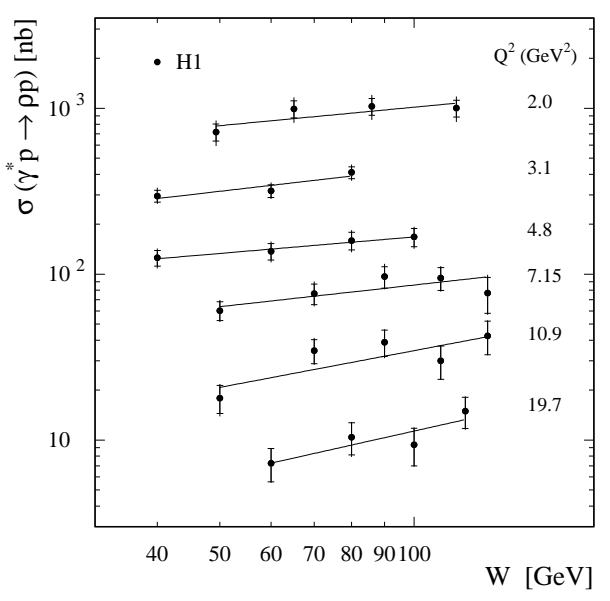

FIGURE 2. The cross section $\sigma_{\gamma^{\star} p \rightarrow \rho p}$ as a function of $W$, for several values of $Q^{2}$. The inner error bars are statistical and the full error bars include the systematic errors added in quadrature. The lines correspond to a fit of the form $\sigma_{\rho} \propto W^{\delta}$.

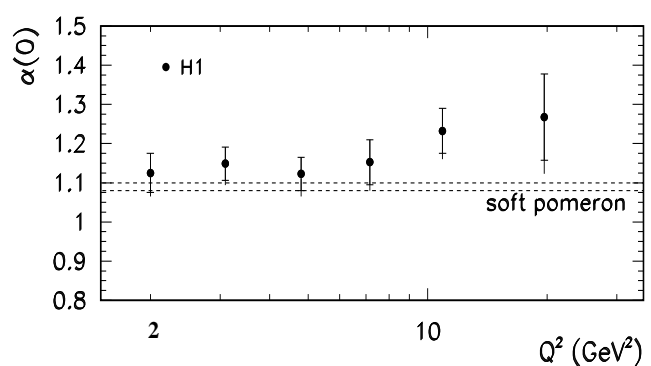

FIGURE 3. The $Q^{2}$ dependence of the intercept $\alpha_{\mathbb{P}}(0)$. The inner error bars are the statistical and non-correlated systematic uncertainties and the outer error bares include the variation of the intercept $\alpha_{\mathbb{P}}(0)$ when assuming $\alpha_{\mathbb{P}}^{\prime}=0$, added in quadrature. The dashed lines represent the range of values obtained for the "soft Pomeron" intercept [23].

The comparison between the measurements of $\sigma_{J / \psi}$ in photoproduction and various pQCD calculations $[19,36]$, based on different gluon distributions, is shown in Fig. 4 [37]. The pQCD calculations are known to suffer from large theoretical uncertainties, therefore no discrimination between the gluon distributions can be made from this comparison. Also shown in Fig. 4 is the result of a calculation [38] based on the Golec-Biernat Wüsthoff (GBW) model [39] of the photon, whose parameters were fitted to the measurements of the inclusive structure function. The GBW model gives a good representation of the data, assuming a double Gaussian wave function of the $J / \psi$.

Although the $W$ dependence of $J / \psi$ photoproduction cannot be explained by the exchange of a universal soft Pomeron, the data can be fitted to the two-Pomeron model [40] as shown in Fig. 5 [41]. The parameters of the established soft and the proposed hard Pomeron trajectories are $\left(\alpha(0), \alpha_{\mathbb{P}}^{\prime}\right)_{\text {soft }}=\left(1.08,0.25 \mathrm{GeV}^{-2}\right)$ and $\left(\alpha(0), \alpha_{\mathbb{P}}^{\prime}\right)_{\text {hard }}=\left(1.418,0.1 \mathrm{GeV}^{-2}\right)$. The relative contributions of the hard, soft and mixing terms of the model are found to vary between $0.1: 0.5: 0.4$ at $W=30 \mathrm{GeV}$ to $0.5: 0.1: 0.4$ at $W=250 \mathrm{GeV}$.

In Fig. 6 the $W$ dependence of $\sigma_{J / \psi}$ is shown for various $Q^{2}$ values [42]. The measurements are compared with theoretical predictions of Frankfurt et al. (FKS) [43] 


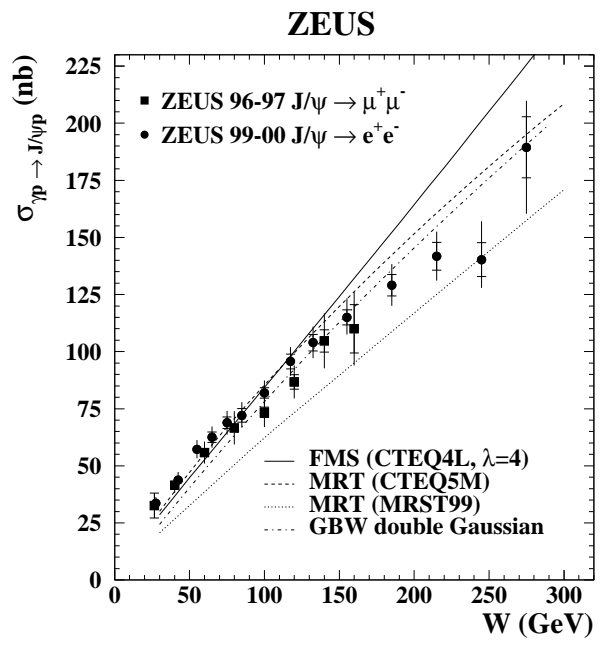

FIGURE 4. The exclusive $J / \psi$ photoproduction cross section as a function of $W$. The inner bars indicate the statistical uncertainties, the outer bars are the statistical and systematic uncertainties added in quadrature. The lines are predictions of pQCD calculations.

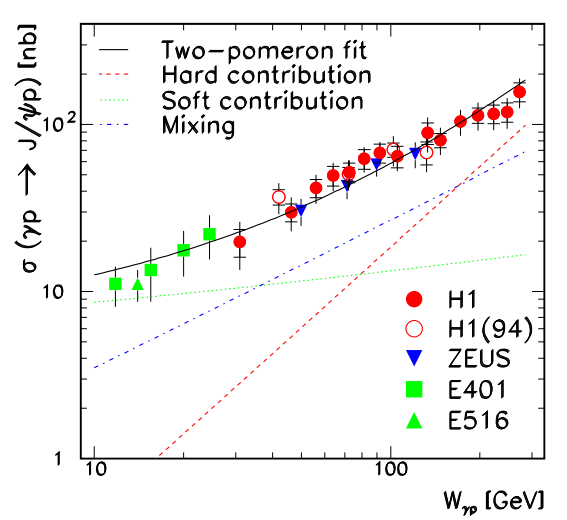

FIGURE 5. The cross section $\sigma(\gamma p \rightarrow J / \psi p)$ versus $W$. The full line is the prediction of the two-Pomeron model by Donnachie and Landshoff. The separate contributions of the hard, soft and mixing terms are also indicated.

using CTEQ4M [44], and of Martin et al. (MRT) [36] using the CTEQ5M [7] parametrization. The calculations are consistent with the data within the uncertainty of the measurements. Also shown in Fig. 7 are the values of $\delta$, obtained by fitting in each $Q^{2}$, the functional form $\sigma_{J / \psi} \propto W^{\delta}$. The results may indicate a slight increase in $\delta\left(Q^{2}\right)$ at high $Q^{2}$.

\section{A Test of SU(4) relation}

The H1 collaboration [16] observed that when the cross sections for various Vs, weighted by the appropriate $S U(4)$ factors $\left(\rho^{0}: \omega: \phi: J / \psi=9: 1: 2: 8\right)$ were plotted as function of $Q^{2}+M_{V}^{2}$, at $W=75 \mathrm{GeV}$, they seem to line up on a universal curve, as shown in Fig. 8. However there was a slight indication that the $J / \psi$ cross sections lie above the curve (see the insert in Fig. 8). The new precise measurements $[37,42]$ of $J / \psi$ production do not fit this picture, as shown in Fig. 9, where the $W$ dependence of light and heavy Vs, appropriately weighted by the $S U$ (4) factor, is plotted in bins of $Q^{2}+M_{V}^{2}$. The $J / \psi$ data lie far above the light $\mathrm{Vs}$ at all scales. It seems, therefore, that the variable $Q^{2}+M_{V}^{2}$ is a good variable for comparing the light $\mathrm{Vs}$ but is not adequate when the $J / \psi$ is added to the comparison. 
ZEUS

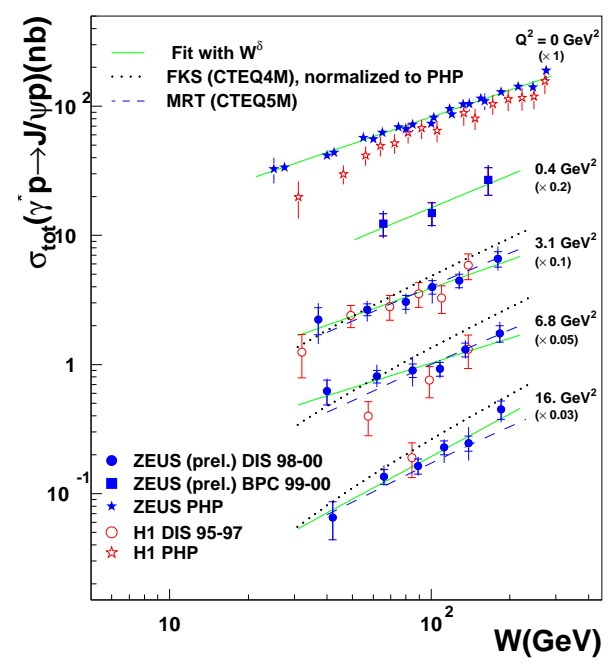

FIGURE 6. $W$ dependence of the cross section $\sigma\left(\gamma^{*} p \rightarrow J / \psi p\right)$ compared with theoretical predictions for $Q^{2}=3.1,6.8$ and $16 \mathrm{GeV}^{2}$. The solid lines indicate the results of fits to the function $W^{\delta}$. The measurements from photoproduction are also shown for comparison.

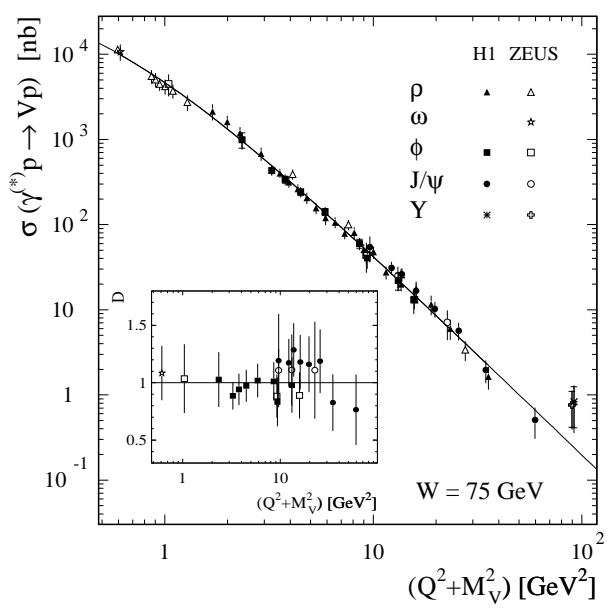

FIGURE 8. The total cross sections for elastic V production scaled by $S U(5)$ factors, as a function of $\left(Q^{2}+M_{V}^{2}\right)$ at $W=75 \mathrm{GeV}$. The curve corresponds to a fit to the H1 and ZEUS $\rho^{0}$ data. The insert shows the deviation of the parameterization from the data for $\omega, \phi$ and $J / \psi$.

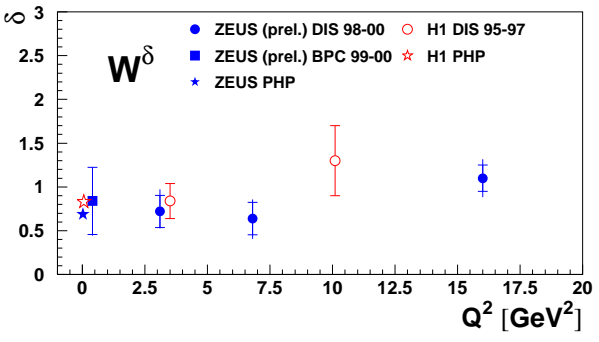

FIGURE 7. The value of $\delta$ plotted as a function of $Q^{2}$ obtained from fits to the form $\sigma_{J / \psi} \propto W^{\delta}$.

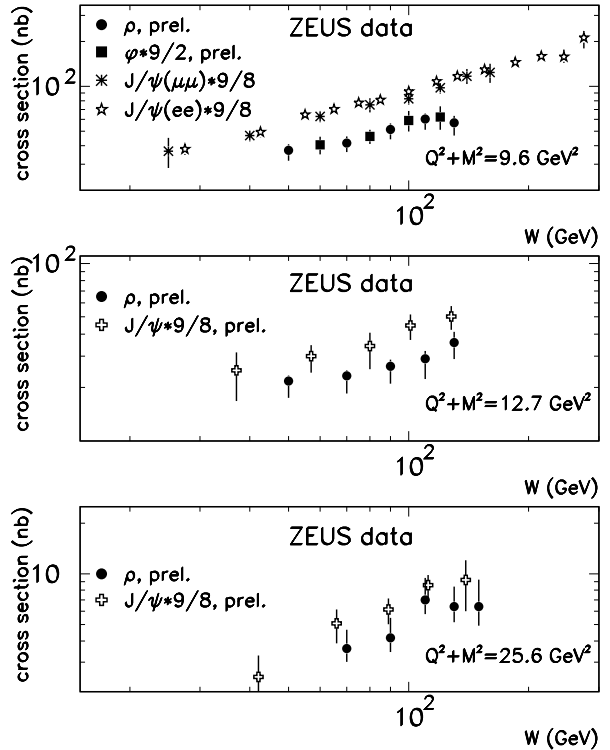

FIGURE 9. Comparison of $\mathrm{V}$ weighted cross section values at fixed $Q^{2}+M_{V}^{2}$ scales, as indicated in figure. 


\section{B Measurement of the Pomeron trajectory, $\alpha_{\mathbb{P}}(t)$}

The $\mathbb{P}$ trajectory, $\alpha_{\mathbb{P}}(t)$, can be measured by fitting the $W$ dependence of the $\gamma^{*} p$ cross section in bins of $t,\left.\frac{d \sigma}{d t}\right|_{t}(W) \sim W^{\delta}$, where $\delta=4\left(\alpha_{\mathbb{P}}(t)-1\right)$. The parameters of the $\mathbb{P}$ trajectory were extracted from measurements of $\rho^{0}[45,46]$ and $J / \psi$ production $[16,37]$ and are summarized in table 1 . The measurements of $\alpha_{\mathbb{P}}(t)$ and the fitted trajectories are shown in Fig. 10. and in Fig. 11

The measured trajectories change with the $M_{V}^{2}$ and $Q^{2}$.

Table 1. Compilation of results obtained for $\alpha_{\mathbb{P}}(0)$ and $\alpha_{I P}^{\prime}$.

\begin{tabular}{|l|c|c|}
\hline$V$ & $\alpha_{\mathbb{P}}(0)$ & $\alpha_{\mathbb{P}}^{\prime}\left(\mathrm{GeV}^{-2}\right)$ \\
\hline$\rho^{0},\left(Q^{2} \simeq 0\right)$ & $1.096 \pm 0.021$ & $0.125 \pm 0.038$ \\
\hline$J / \psi(\mathrm{H} 1)$ & $1.27 \pm 0.05$ & $0.08 \pm 0.017$ \\
\hline$J / \psi($ ZEUS $)$ & $1.200 \pm 0.009_{-0.010}^{+0.004}$ & $0.115 \pm 0.018_{-0.015}^{+0.008}$ \\
\hline$\rho^{0}$ (ZEUS, DIS $)$ & $1.14 \pm 0.01_{-0.03}^{+0.03}$ & $0.04 \pm 0.07_{-0.04}^{+0.13}$ \\
\hline
\end{tabular}

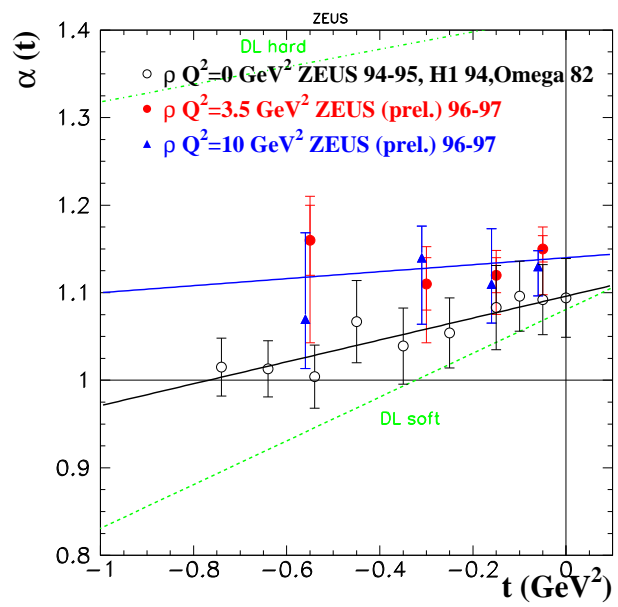

FIGURE 10. $\alpha_{\mathbb{P}}(t)$, as measured for DIS $\rho^{0}$, for two $Q^{2}$ bins, $2<Q^{2}<6$ and $6<Q^{2}<40 \mathrm{GeV}^{2}$, compared to $\rho^{0}$ photoproduction results. The solid lines are a fit to the data.

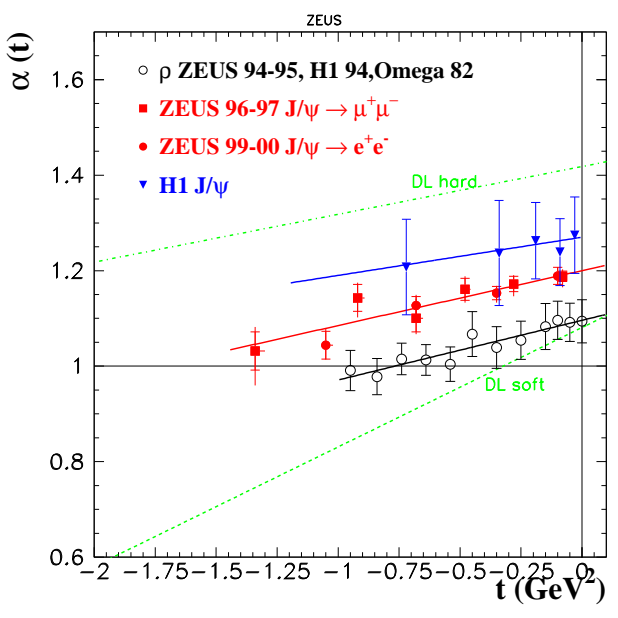

FIGURE 11. The value of $\alpha_{\mathbb{P}}(t)$ as a function of $t$ as measured for, $J / \psi$ photoproduction compared with previous results for $\rho^{0}$ photoproduction.

\section{ANGULAR DISTRIBUTIONS AND HELICITY STUDIES}

The separation of the $\gamma^{*} p$ cross section into the contribution from longitudinal photons, $\sigma_{L}$, and transverse photons, $\sigma_{T}$, can be performed by measuring the $\mathrm{V}$ 
density matrix elements, $r_{i j}^{\alpha}[30]$. These matrix elements are used to parameterize the angular distribution $W\left(\cos \theta_{h}, \phi_{h}, \Phi_{h}\right)$ of the helicity angles. The density matrix elements, $r_{i j}^{\alpha}$, are related to the transition amplitudes of longitudinal or transverse photons into longitudinal or transverse Vs. The transition amplitudes are denoted as $T_{i j}$, where $i=0(1)$ is a transverse (longitudinal) photon going into a $j=0(1)$ transverse (longitudinal) V. The relations between the transition amplitudes have been predicted by pQCD models $[13,31,32]$.

\section{A The ratio of longitudinal to transverse cross sections,$$
R=\sigma_{L} / \sigma_{T}
$$

The most extensive measurements $[28,46]$ of $R\left(Q^{2}\right)$ were preformed for $\rho^{0}$ electroproduction. The ratio $R$ of cross sections induced by longitudinal polarized virtual photons to the transversely polarized ones, $\sigma_{L} / \sigma_{T}$, is related to the spin density matrix elements of $\rho^{0}$ through

$$
R=\frac{1}{\epsilon} \frac{r_{00}^{04}-\Delta^{2}}{1-\left(r_{00}^{04}-\Delta^{2}\right)},
$$

where $r_{00}^{04}$ is a linear combination of the $\rho^{0}$ density matrix elements, and $\Delta^{2}$ is proportional to the contribution of the $\mathrm{SCH}$ non Conservation amplitude over the total amplitude. A small breaking of SCHC has been predicted [13] and measured [28,29] $(\Delta=7.9 \pm 1.6 \%[29])$.

The $Q^{2}$ and $W$ dependence of $R$ is plotted in Fig. 12 and Fig. 13, respectively. A continuous rise of $R$ with $Q^{2}$ is observed. The $Q^{2}$ dependence of $R$ is well described by a functional form $R=\frac{1}{\xi}\left(Q^{2} / M_{\rho}^{2}\right)^{\kappa}$ with $\xi=2.16 \pm 0.05$ and $\kappa=0.74 \pm 0.02$ [46]. On the other hand, there seems to be no $W$ dependence of $R$ for fixed values of $Q^{2}$. This indicates that $\sigma_{L}$ and $\sigma_{T}$ have the same energy dependence, which is fixed by the $Q^{2}$ value rather than the longitudinal or transverse configuration.

\section{B A measurement of the $t$ dependence of the helicity structure}

The extracted [47] values of $r_{00}^{04}$ for $\rho^{0}$ production are presented in Fig. 14, where

$r_{00}^{04} \propto \frac{\left|T_{00}\right|^{2}+\left|T_{01}\right|^{2}}{N} \propto \sigma_{L} /\left(\sigma_{T}+\sigma_{L}\right)$ and $N=\sum_{i, j}\left|T_{i j}\right|^{2}$. No significant variation of $r_{00}^{04}$ with $t^{\prime}=\left|t-t_{\text {min }}\right|$ is observed. This observation implies that the slopes of the exponentially falling $t$ distributions for the transverse and longitudinal $s$-channel helicity conserving amplitudes, $T_{00}$ and $T_{11}$, are very similar.

The combination $r_{00}^{5}+2 r_{11}^{5}$ is presented in Fig. 15 [47]. $r_{00}^{5}$ is expected [13,31,32] to be proportional to the product of the dominant non-flip amplitude $T_{00}$ and the single flip $T_{01}$ amplitude, $r_{00}^{5} \propto \frac{1}{N} \operatorname{Re}\left(T_{00} T_{01}\right)$. The $T_{01}$ amplitude is expected to be the largest helicity flip amplitude. The $r_{11}^{5}$ matrix element, on the other hand is 


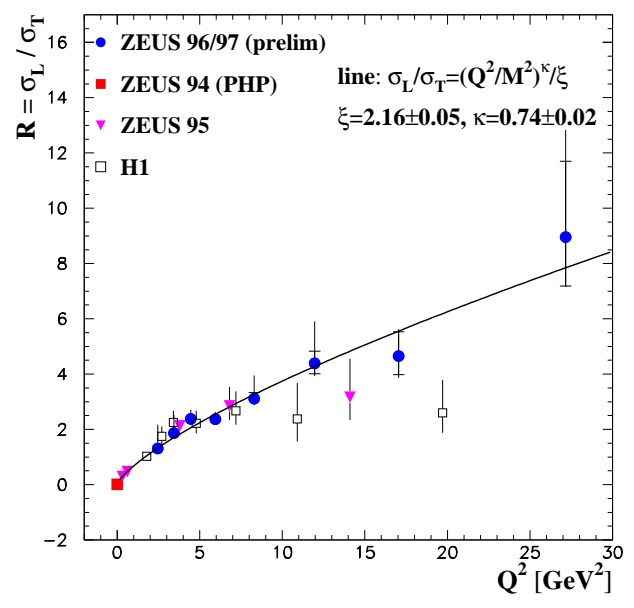

FIGURE 12. The values of $R$ as a function of $Q^{2}$.

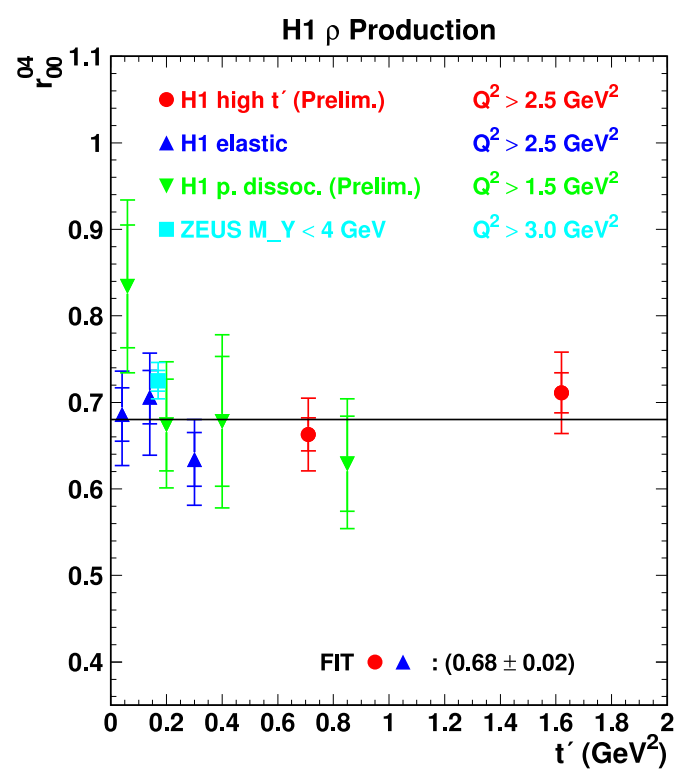

FIGURE 14. Measurement of $r_{00}^{04}$ as a function of $t^{\prime}$. The curve is a result of fitting the data with calculation of [13].

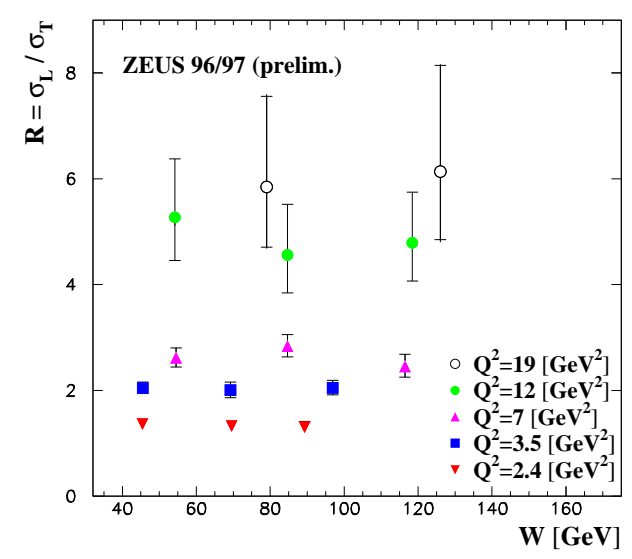

FIGURE 13. The value of $R$ is presented as a function of $W$, for different $Q^{2}$ values.

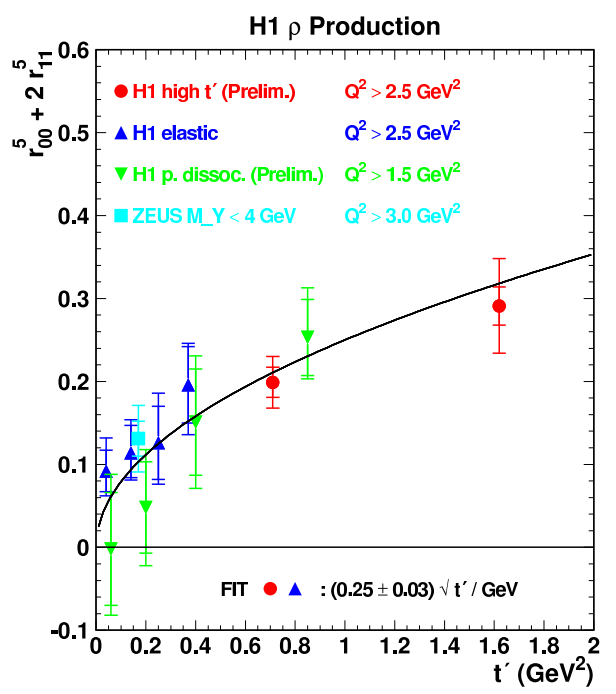

FIGURE 15. Measurement of $r_{00}^{5}+2 r_{11}^{5}$ as a function of $t^{\prime}$. The curve is a result of fitting the data with calculation of [13]. For SCHC a null result is expected, independent of $t^{\prime}$.

proportional to the non-dominant amplitudes $r_{11}^{5} \propto \frac{1}{N} R e\left(2 T_{11} T_{10}^{\dagger}-2 T_{10} T_{1-1}^{\dagger}\right)$. The strong $t^{\prime}$ dependence of the $r_{00}^{5}+2 r_{11}^{5}$ combination is thus attributed mainly to the predicted $[13,31,32] \sqrt{t^{\prime}}$ dependence of the ratio of $T_{01}$ to the non-flip amplitudes.

The values for $r_{00}^{1}+2 r_{11}^{1}$ are shown in Fig. 16. The matrix element $r_{00}^{1}$ is proportional to the single flip amplitude $T_{01}$ square, $r_{00}^{1} \propto-\frac{\left|T_{01}\right|^{2}}{N} \propto(\sqrt{t})^{2}$, while the matrix element $r_{11}^{1}$ is expected to have the same $t$ dependence as the double flip amplitude $T_{1-1}, r_{11}^{1} \propto \frac{1}{N}\left(T_{11} T_{1-1}^{\dagger}\right) \propto t$, therefore the $t^{\prime}$ dependence of the combination $r_{00}^{1}+2 r_{11}^{1}$ is expected to be linear, up to effects of the single and double-flip 
amplitudes in the denominator $N$. The combination $r_{00}^{1}+2 r_{11}^{1}$ is significantly different from zero and negative, which implies violation of SCHC. The sign of the combination gives information on the relative strength of the $T_{01} T_{01}^{\dagger}$ and $T_{11} T_{1-1}^{\dagger}$ products of amplitudes, $\left|T_{01}\right|^{2}>\left(T_{11} T_{1-1}^{\dagger}\right)$, and therefore $T_{01}>T_{1-1}$. It confirms that the $T_{01}$ amplitude is significantly larger than the double flip amplitude in the present kinematic domain.

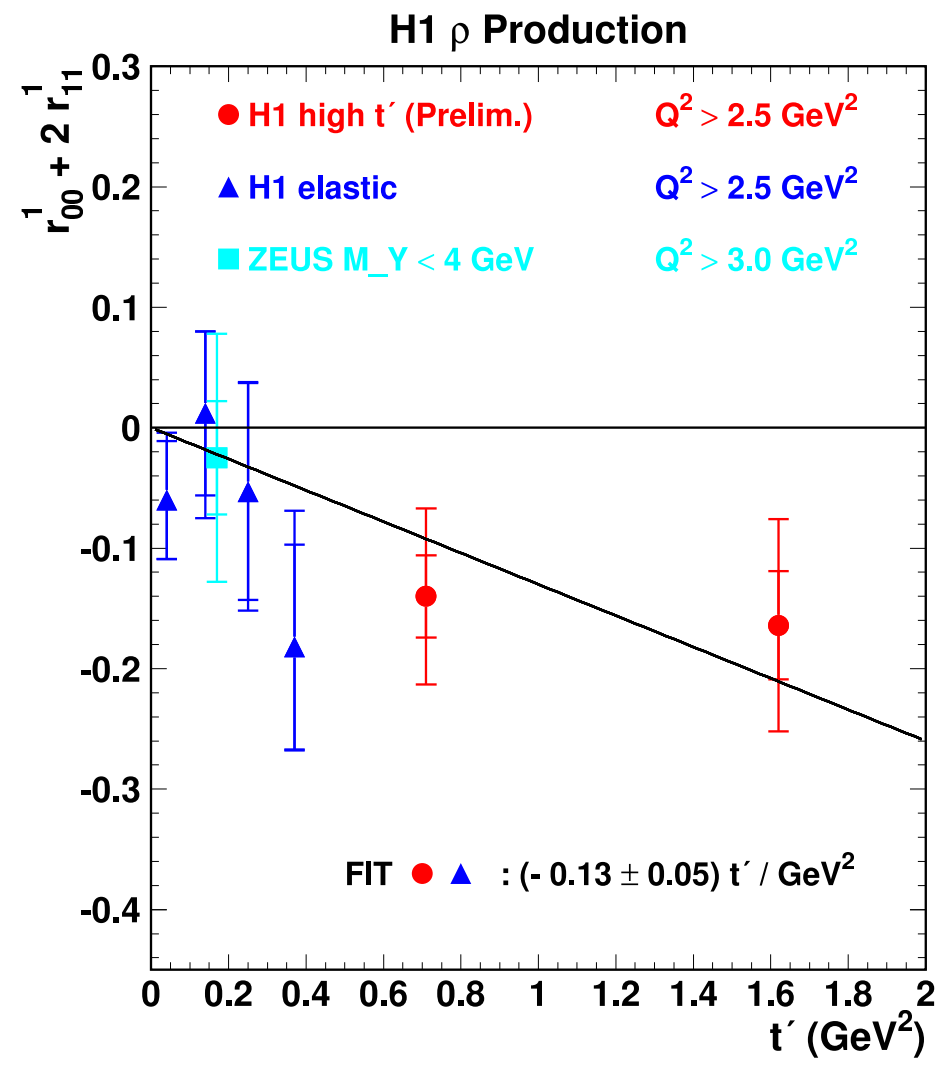

FIGURE 16. Measurement of $r_{00}^{1}+2 r_{11}^{1}$ as a function of $t^{\prime}$. The curve is a result of fitting the data with calculation of [13]. For SCHC a null result is expected, independent of $t^{\prime}$.

\section{THE DIFFERENTIAL CROSS SECTION AT LOW $t$}

The differential cross section as function of $t$ has been studied for $\rho^{0}$ electroproduction. The values of $b\left(Q^{2}\right)$ are extracted from a fit to an exponential form, $\frac{d \sigma}{d t} \propto \exp (b t)$, for $t<1 \mathrm{GeV}^{2}$ in elastic production $\left(b_{e l}\right)$ and for $t<2 \mathrm{GeV}^{2}$ in proton dissociation $\rho^{0}$ production $\left(b_{p d}\right)$, for different $Q^{2}$ regions . The data cover 
a kinematic range of $50<W<140 \mathrm{GeV}$ and $2<Q^{2}<80 \mathrm{GeV}^{2}$ (elastic) and $2<Q^{2}<50 \mathrm{GeV}^{2}$ (proton dissociation).

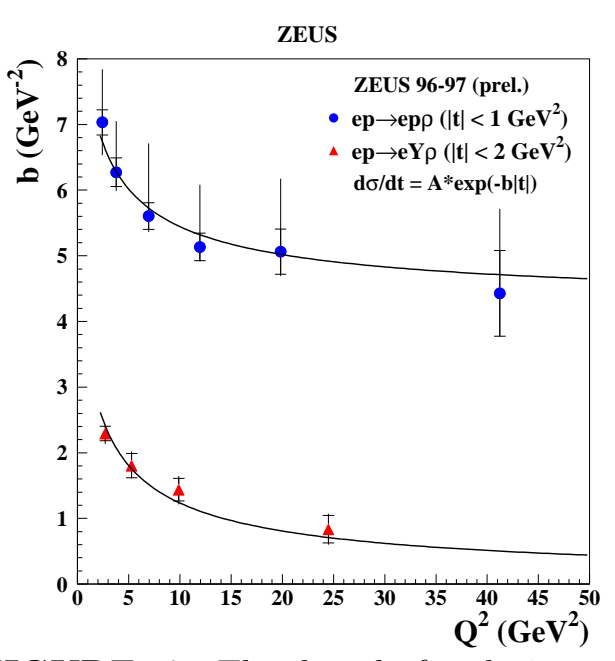

FIGURE 17. The slope $b$ of exclusive and proton-dissociative electroproduction of $\rho^{0}$.

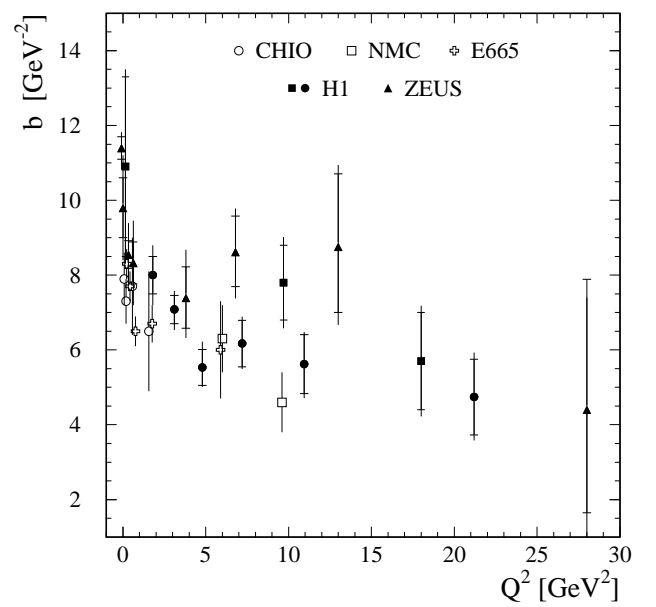

FIGURE 18. Exponential slope $b$ of the $t$ dependence for elastic $\rho^{0}$ production as a function of $Q^{2}$.

The values of $b_{e l}$ and $b_{p d}$ are shown in Fig. 17 as a function of $Q^{2}$. The $Q^{2}$ dependence of $b$ is well described by the following functional forms,

$$
\begin{aligned}
b_{p d}\left(Q^{2}\right) & =A /\left(M_{V}^{2}\left(1+R\left(Q^{2}\right)\right)\right), \\
b_{e l}\left(Q^{2}\right) & =b_{p d}\left(Q^{2}\right)+b_{\infty}, \\
R=\frac{\sigma_{L}}{\sigma_{T}} & =\frac{1}{\xi}\left(Q^{2} / M_{V}^{2}\right)^{\kappa} \\
A & =3.46 \pm 0.14 \\
b_{\infty} & =4.21 \pm 0.12 \mathrm{GeV}^{-2} .
\end{aligned}
$$

One clearly sees the decrease in the value of $b_{e l}$ with $Q^{2}$, approaching an asymptotic value, from fit $b_{\infty}=4.21 \pm 0.12 \mathrm{GeV}^{-2}$. The slopes $b_{e l}$ and $b_{p d}$ are described with the same $Q^{2}$ dependence, in compliance with the expectations of vertex factorization [34]. It is interesting to note that the ad hoc parametrization of $b_{p d}$, based on the $Q^{2}$ dependence of $R$, describes well the measurements of $b_{p d}$ and $b_{e l}$. The parameterization is motivated by the expected $Q^{2}$ dependence of the cross section and the relation between the probability for longitudinal configuration and the size of the $q \bar{q}$ pair.

The measurements of $b_{e l}$, done by $\mathrm{H} 1$ [28] (Fig. 18), show the same $Q^{2}$ behavior. It is also evident that at low $Q^{2}$, the HERA measurements lie systematically above the low energy fixed target results. This might indicate shrinkage of the diffractive peak, at low $Q^{2}$, as $W$ increases.

In order to test the factorization hypothesis at the proton vertex [34], the ratio of $d \sigma / d t$ of the elastic and proton dissociation reactions is studied as function of $Q^{2}$, for two fixed values of $t$, as shown in Fig. 19. The ratio is consistent with being 
$Q^{2}$ independent. This lends support to the hypothesis that factorization holds at the proton vertex for $\rho^{0}$ electroproduction in the region $2<Q^{2}<20 \mathrm{GeV}^{2}$ and $|t|<0.4 \mathrm{GeV}^{2}$.

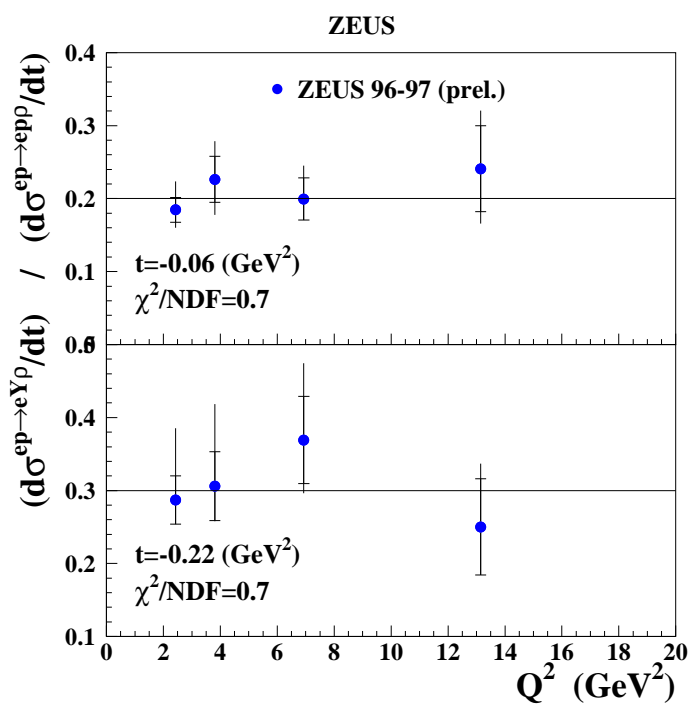

FIGURE 19. Ratio of proton-dissociative and exclusive $\rho^{0}$ electroproduction processes as function of $Q^{2}$ for fixed $t$ values, as given in the figure. The lines are the results of a best fit to a constant ratio at each $t$ value.

\section{CONCLUSIONS}

It is evident that the hardness of the process in the elastic production of vector mesons in ep collisions at high energy, is influenced by both initial (e.g. $Q^{2}$ ) and final state configurations (e.g. $M_{V}^{2}$ ). This is demonstrated by the change of the energy dependence with $Q^{2}$ and $M_{V}^{2}$, and by the decrease of the $b$ slope with increasing $Q^{2}$ and $M_{V}^{2}$. Longitudinal and transverse photons seem to have the same energy dependence at fixed $Q^{2}$, as well as the same $t$ dependence. This indicates that the $q \bar{q}$ pairs initiated by a longitudinal or transverse photon, and contributing to the production of a given vector meson, are typically of the same size. This size is determined both by the virtualty $Q^{2}$ of the fluctuating photon and by the probability to form the vector meson final state.

Although the overall picture is consistent with pQCD predictions, the quantitative calculations need further improvements. 


\section{ACKNOWLEDGMENTS}

I am very grateful to Alberto Santoro and the LISHEP organizing committee for their financial support and hospitality during the LISHEP advanced school on HEP and the following workshop on diffractive physics.

This research was supported in part by the Israel Science Foundation (ISF), by the German Israel Foundation (GIF) and by Israel Ministry of Science. 


\section{REFERENCES}

1. ZEUS Collab., M. Derrick et al., Phys. Lett. B316 (1993) 412;

Zeit. Phys. C65 (1995) 379.

2. H1 Collab., I. Abt et al., Nucl. Phys. B407 (1993) 515.

3. ZEUS Collab., M. Derrick et al., Phys. Lett. B315 (1993) 481.

4. H1 Collab., I. Abt et al., Nucl. Phys. B429 (1994) 477.

5. V.N. Gribov and L.N. Lipatov, Sov. J. Nucl. Phys. 15 (1972) 438and 675;

G. Altarelli and G. Parisi, Nucl. Phys. 126 (1977) 297;

Yu.L. Dokshitzer, Sov. Phys. JEPT 46 (1977) 641.

6. ZEUS Collab., J. Breitweg et al., Eur. Phys. J. C7 (1999) 609;

H1 Collab., S. Aid et al., Nucl. Phys. B470 (1996) 3;

A.D. Martin et al., Eur. Phys. J. C14 (2000) 133;

M. Gluck E. Reya and A. Vogt Eur. Phys. J. C5 (1998) 461.

7. CTEQ Collab. H.L. Lai et al., Eur. Phys. J. C12 (2000) 375.

8. S.J. Brodsky, L. Frankfurt, J.F. Gunion, A.H. Mueller, and M. Strikman, Phys. Rev. D50 (1994) 3134.

9. H. Abramowicz, L. Frankfurt and M. Strikman, Surveys High Energy Phys. 11 (1997) 51.

10. L. Frankfurt, M. McDermott, V. Guzey and M. Strikman, Eur. Phys. J. C16 (2000) 641.

11. P.D.B. Collins, An Introduction to Regge Theory and High Energy Physics, Cambridge University Press, England, (1997).

12. L. Frankfurt, W. Koepf and M. Strikman, Phys. Rev. D54 (1996) 3194;

M.G. Ryskin, Zeit. Phys. C57 (1993) 89;

A.D. Martin, M.G. Ryskin and T. Teubner, Phys. Rev. Lett. B454 (1999) 399.

13. D.Y. Ivanov and R. Kirschner, Phys. Rev. D58 (1998) 114026.

14. ZEUS Collab., paper submitted to the 29th International Conference on High Energy Physics, Abstract 793, Vancouver, (1998);

ZEUS Collab., J. Breitweg et al., Zeit. Phys. C75 (1997) 215;

ZEUS Collab., J. Breitweg et al., Phys. Lett. B487 (2000) 273.

15. ZEUS Collab., paper submitted to the 30th International Conference on High Energy Physics, Abstract 880, Osaka, (2000).

16. H1 Collab., C. Adloff et al., Phys. Lett. B483 (2000) 360.

17. ZEUS Collab., J. Breitweg et al., Eur. Phys. J. C6 (1993) 603.

18. A.V. Radyushkin, Phys. Rev. D56 (1997) 5524;

X.Ji, J. Phys. G. G24 (1998) 1181..

19. L. Frankfurt, M. McDermott and M. Strikman, Journal of High Energy Phys. 103 (2001) 45.

20. See e.g. M.F. McDermott, DESY-00-126, (2000), and references therein.

21. Gribov V N, JETP Lett. 41 (1961) 667.

22. K. Kang, J.R. Cudell, V.V. Ezhela, S.B. Lugovsky and N.P. Tkachenko, hep$\mathrm{ph} / 9812429$.

23. A. Donnachie and P.V. Landshoff, Nucl. Phys. B231 (1984) 189;

A. Donnachie and P.V. Landshoff, Phys. Lett. B296 (1992) 227. 
24. H1 Collab., C. Adloff et al., Nucl. Phys. B497 (1997) 3.

25. ZEUS Collab., J. Breitweg et al., Phys. Lett. B443 (1998) 394.

26. A. Levy, Phys. Lett. B424 (1998) 191.

27. ZEUS Collab., paper submitted to the International Europhysics Conference on High Energy Physics, Abstract 548, Budapest, 2001.

28. H1 Collab., C. Adloff et al., Eur. Phys. J. C13 (2000) 371.

29. ZEUS Collab., J. Breitweg et al., Eur. Phys. J. C12 (2000) 393.

30. K. Schilling and G. Wolf,Nucl. Phys. B61 (1973) 381.

31. I. Royen and J. Cudell, Nucl. Phys. B545 (1999) 505;

Phys. Lett. B513 (2001) 337.

32. E. Kuraev, N. Nikolaev and B. Zakharov, JETP Lett. 68 (1998) 696..

33. M.G. Ryskin, Yu.M. Shabelski and A.G. Shuvaev, Phys. Lett. B446 (1999) 48.

34. V.N. Gribov, Phys. Rep. 101 (1983) 169.

35. D. Brown for H1 Collab., Proceeding of DIS01, 27 April - 1 May 2001, Bologna, Italy.

36. A. D. Martin, M. G. Ryskin and T. Teubner, Phys. Rev. D62 (2000) 014022.

37. ZEUS Collab., S. Chekanov et al,. Europ. Phys. Journal C; DOI 10.1007/s10052002-0953-7 (Online Publication).

38. A.C Caldwell and M.S. Soares, Nucl. Phys. A696 (2001) 125.

39. K. Golec-Biernat and M. Wüsthoff, Phys. Rev. D59 (1999) 014017;

K. Golec-Biernat and M. Wüsthoff, Phys. Rev. D60 (1999) 114023.

40. A. Donnachie, P.V. Landshoff, Phys. Lett. B437 (1998) 408.

41. H1 Collab., C. Adloff et al., Phys. Lett. B483 (2000) 23

42. A. Levy for the ZEUS Collaboration, presented at DIS02, 28 April - 3 May 2001.

43. L. Frankfurt, W. Koepf and M. Strikman, Phys. Rev. D57 (1998) 512.

44. H.L. Lai et al., Phys. Rev. D55 (1997) 1280.

45. ZEUS Collab.; J. Breitweg et al., Eur. Phys. J. C14 (2000) 2213.

46. A. Kreisel for ZEUS collaboration, Proceeding of DIS01, 27 April - 1 May 2001, Bologna, Italy.

47. H1 Collab., C. Adloff et al., accepted by Phys Lett. B , 2002. 